\title{
Review Article \\ Safety of Chinese Herbal Medicine for Chronic Obstructive Pulmonary Disease
}

\author{
Meaghan Coyle, ${ }^{1}$ Johannah Linda Shergis, ${ }^{1}$ Shaonan Liu, ${ }^{2,3}$ Lei Wu, ${ }^{2,3}$ Anthony Lin Zhang, \\ Xinfeng Guo, ${ }^{2,3}$ Chuanjian Lu, ${ }^{2,3}$ and Charlie Changli Xue ${ }^{1,4}$ \\ ${ }^{1}$ Traditional \& Complementary Medicine Research Program, School of Health Sciences and \\ Health Innovations Research Institute (HIRi), RMIT University, Bundoora, Melbourne, VIC 3083, Australia \\ ${ }^{2}$ Guangdong Provincial Hospital of Chinese Medicine, Guangzhou 510120, China \\ ${ }^{3}$ The Second Clinical College, Guangzhou University of Chinese Medicine, Guangzhou 510405, China \\ ${ }^{4}$ Guangdong Provincial Academy of Chinese Medical Sciences, Guangzhou 510120, China
}

Correspondence should be addressed to Meaghan Coyle; meaghan.coyle@rmit.edu.au

Received 7 December 2014; Accepted 10 March 2015

Academic Editor: Chang-Gue Son

Copyright (C) 2015 Meaghan Coyle et al. This is an open access article distributed under the Creative Commons Attribution License, which permits unrestricted use, distribution, and reproduction in any medium, provided the original work is properly cited.

Chinese herbal medicine (CHM) is increasingly used by patients with chronic obstructive pulmonary disease (COPD); however, there has been no systematic evaluation of its safety. This review examined the adverse events (AEs) reported in clinical studies of CHM for COPD. Five English databases (PubMed, Embase, CINAHL, AMED, and CENTRAL) and four Chinese databases (CBM, CNKI, CQVIP, and Wanfang Data) were searched from inception to May 2013. Adverse event data, including nature, severity, author-assigned causality, management, and outcome, were extracted from included studies. Descriptive statistics were used for the rate of adverse events. Of the 152 included studies, AEs were reported in 47 studies. The rate of adverse events was slightly lower in the CHM groups compared with controls (84 events in 5,909 participants, $1.4 \%$ versus 102 events in 5,676 participants, $1.8 \%$ ). The most frequently reported adverse event was nausea (28 cases in the CHM groups and 19 cases in the control groups), which was more common in studies where CHM was combined with pharmacotherapy to treat acute exacerbation of COPD. Other frequent adverse events were abdominal discomfort, dry mouth, and dizziness. Detailed information about the adverse events was scant. Overall, CHM appears to be well tolerated in people with COPD.

\section{Introduction}

Chronic obstructive pulmonary disease (COPD) is a persistent disease with recurrent daily symptoms. Despite available drug treatments such as bronchodilators and steroids, 20$43 \%$ of people with COPD also use complementary therapies, including Chinese herbal medicine (CHM), to alleviate their symptoms [1,2]. The frequent use of CHM by COPD patients may be underpinned by their belief that herbal medicines are safe $[1,3]$. Patients taking complementary therapies felt that these therapies did not have risks because of their "naturalness" [1].

CHM appears to have clinically beneficial effects with very low risk of adverse events. Recent systematic reviews demonstrated that CHM improved lung function [4] and quality of life [5]. While many of the studies included in these reviews did not report adverse events (AEs), a number of studies included information concerning minor events. Therefore, these authors concluded that CHM was overall well tolerated by COPD patients $[4,5]$.

Without specific reference to COPD, one prospective study on general safety of a range of CHM interventions over a four-week period showed that $14 \%$ of patients reported an $\mathrm{AE}$ which was associated with $\mathrm{CHM}[6]$. The most common AE was diarrhoea (4.2 events per 100 patients over 4 weeks). Hong Kong Hospital data revealed that, in an eight-month period, $0.2 \%$ of admissions were associated with $\mathrm{AE}$ from CHM [7]. The AEs were mostly minor; however, some were severe. Several reviews of AEs associated with CHMs have described the nature of the $\mathrm{AE}$, but not the number of events $[3,8-10]$, often due to omissions in included studies. Finally, one study reported one adverse event per 633 consultations 
with a CM practitioner (for either CHM treatment or acupuncture) [11].

Due to the wide ranging CHM formulae used for COPD and the generic term "Chinese herbal medicine" not commonly used, combined with variability in publication keywords, it is difficult to identify relevant studies through electronic database searching. To date, there has been no specific evaluation of the safety of CHM for people with COPD based on clinical research evidence. Therefore, we systematically reviewed AEs reported in clinical studies of CHM for COPD. This review describes the incidence of AEs including nature, severity, and relationship with $\mathrm{CHM}$ treatment and was undertaken as part of a broader review of the evidence of efficacy (reported elsewhere).

\section{Materials and Methods}

The search strategy was guided by the Cochrane Airways Group methodology [12]. Nine databases were searched from inception to May 2013 (English: PubMed, Embase, CINAHL, AMED, and CENTRAL; Chinese: CBM, CNKI, CQVIP, and Wanfang Data). For the broader review that evaluated CHM efficacy for treating COPD, studies were included if they used CHM alone or combined with pharmacotherapy compared to pharmacotherapy, placebo, or no treatment. All forms of CHM were included, such as oral, intravenous, and intramuscular. Studies were eligible if they reported on adverse events in addition to any one of the following outcomes: lung function $\left(\mathrm{FEV}_{1}, \mathrm{FVC}\right)$, symptom severity (dyspnoea scales mMRC scale, Borg scale, and DVAS), exercise capacity (6 MWT), arterial blood gases $\left(\mathrm{PaO}_{2}, \mathrm{PaCO}_{2}\right)$, COPD exacerbations, BODE index, or effective rate. Controlled clinical trials and noncontrolled studies published in English and Chinese were included. Studies which did not report the number of people randomised to the treatment and control groups (for controlled studies) were excluded.

The title and abstract of retrieved citations were screened for eligibility, and full text was retrieved where necessary. Eligibility assessment was confirmed by a second person. Study characteristics and AE data were extracted into a predefined form, with double-data extraction and consistency checking to ensure accuracy of the data. Studies which did not report on AEs were excluded from further analysis. Items relating to AEs included nature of AE and number of events, severity, and author-assigned causality, how the AE was managed, and the outcome. We used only the author-assigned causality (if reported) to avoid introducing bias, as we deemed it unlikely that sufficient information would be reported in the original publications to determine causality. For controlled studies, AEs were described according to group allocation or separately if group allocation was not specified by the study author. Where two events were reported together, for example, nausea and headache, these were considered as one incident for the purposes of this review.

The rate of AEs was calculated overall and according to $\mathrm{CHM}$ or control groups by dividing the number of events by the number of participants, expressed as a percentage. Similar AEs were grouped to allow for reporting of events by broad category. Analysis of time to event and multiple events per participant were planned but can not be performed due to insufficient details.

\section{Results}

One hundred and fifty-two (152) of the 609 identified studies reported on AEs (occurrence or absence) (see Figure 1). The majority of studies were RCTs (144 studies), while two were nonrandomised controlled trials and six were noncontrolled studies. A total of 11,999 participants were included in this analysis, with 6,261 receiving treatment with $\mathrm{CHM}$ and 5,676 people allocated to control groups. The age of participants ranged from 18 to 92 years, and the median of mean age was 65.6 years. Duration of treatment ranged from three days to one year, with a median duration of two weeks. The most common CHM was Tan re qing injection (27 studies).

Adverse events occurred in 47 studies (involving 4,057 participants) [13-59] (see Table 1). A total of 207 events were reported $(11,999$ participants, $1.7 \%)$. In the controlled studies, 84 events occurred in those who received CHM intervention (5,909 participants, 1.4\%) and 102 events in those who were allocated to the control groups $(5,676,1.8 \%)$. Nausea was the most frequently reported AE in both CHM and control groups (occurring in 28 cases and 19 cases, resp.). The vast majority of nausea AEs occurred in people with acute exacerbation of COPD where CHM was combined with pharmacotherapy. Other frequently reported AEs included abdominal discomfort ( 13 cases in CHM, 3 cases in control), dry mouth ( 7 cases in CHM, 9 cases in control), and dizziness ( 4 cases in CHM, 10 cases in control).

For analysis, studies were grouped according to intervention categories: (1) CHM alone or (2) CHM combined with pharmacotherapy and by study design: (1) controlled studies and (2) noncontrolled studies. Events were categorised into three broad groups: gastrointestinal events, dermatological events, and miscellaneous events (see Table 2). The CHM formulae and ingredients in studies where AEs occurred in the CHM groups are described in Table 3.

\subsection{Controlled Studies}

3.1.1. CHM Alone. Eight studies compared CHM to controls and six reported the occurrence of AEs [39, 40, 44, 48, 50, 58]. Two studies reported that no events occurred $[60,61]$. CHM was compared with placebo $[44,60]$, no treatment [58], or pharmacotherapy $[39,40,48,50,61]$. Twenty-nine (29) AEs were reported in the six studies. Thirteen of these AEs occurred in the treatment groups and 16 events in the control groups.

The number of gastrointestinal AEs was lower in those who received $\mathrm{CHM}$ compared with controls (2 AEs versus 6 AEs) but similar for miscellaneous AEs (11 AEs versus 10 AEs) (see Table 2). No dermatological AEs were reported in both the CHM and comparator groups. The most frequently reported event in the CHM groups was shivering/shaking hands ( 3 events), while bloating was the most frequent $\mathrm{AE}$ in the comparator groups (3 events). In studies which described the severity of AEs, all were reported as mild with the exception of three infections which were judged as severe and 


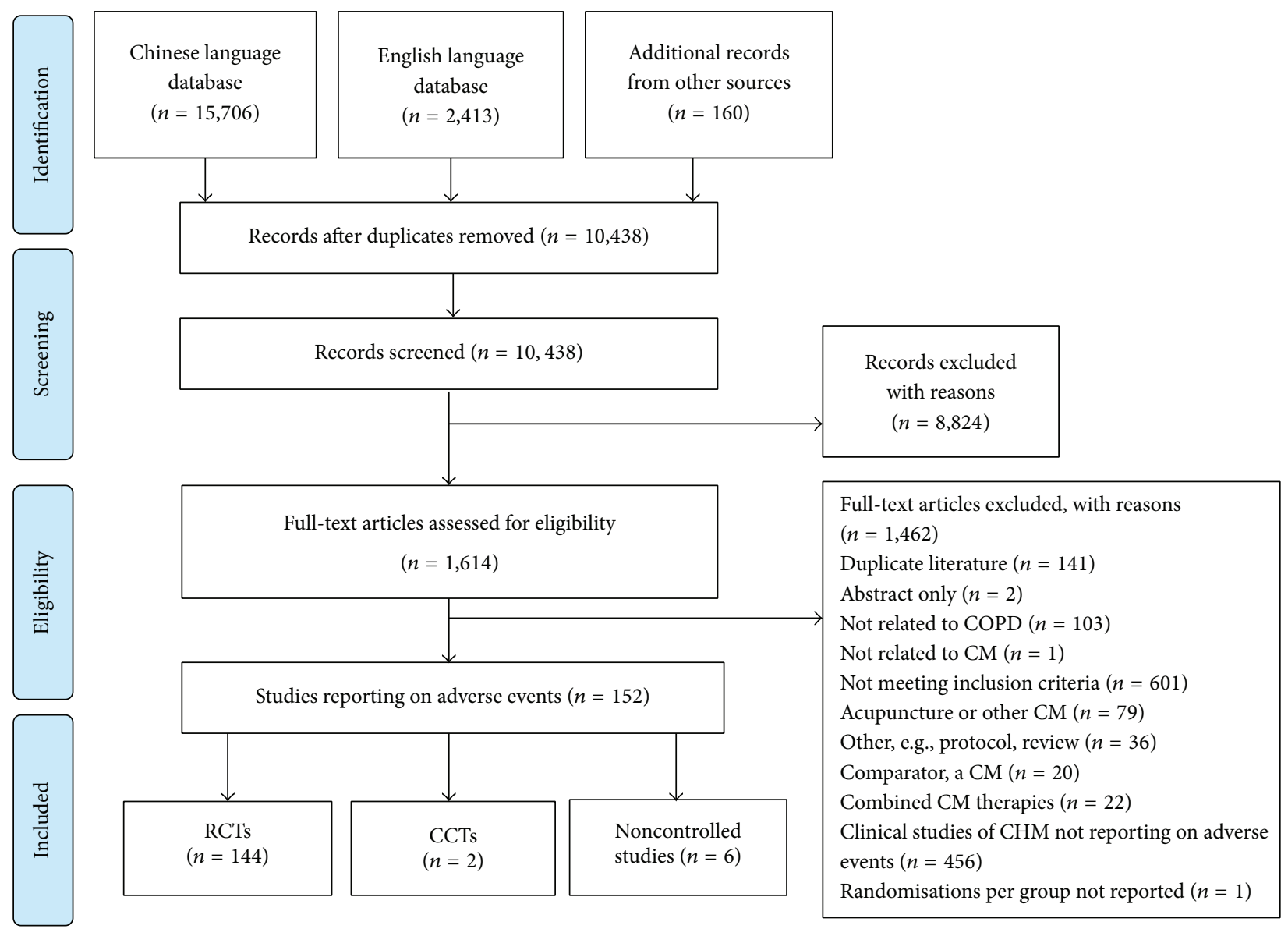

CM: Chinese medicine

CCT: controlled clinical trials

RCT: randomised controlled trials

FIGURE 1: Flow diagram of included studies.

lead to patient death. One death occurred in the treatment group but was deemed unlikely to be related to treatment and two in the control group of the same study [58]. In one study that evaluated Mai men dong tang (Bakumondoto) two cases of elevated alkaline phosphatase (ALP) were considered possibly related to treatment; however, retest was not performed and a full exploration of causality could not be determined [39]. To manage AEs, study investigators changed the CHM dose [50], prescribed other drugs [48], continued treatment as allocated $[48,50]$, or did not administer treatment $[40]$, or the patients withdrew [58].

3.1.2. CHM Plus Pharmacotherapy. One hundred and thirtyeight (138) studies combined CHM with pharmacotherapy, and 37 of these reported the occurrence of 167 AEs [13-30, 32$34,36-38,41-43,45-47,49,51-54,56,57]$. In the intervention group, CHM was given with the same pharmacotherapy as the control group. Eighteen studies did not specify the pharmacotherapy used $[13-16,19,20,23,25,26,29,33,34$, $38,47,49,51,53,54]$. Antibiotics were used in 15 studies, methylxanthines in 14 studies, mucolytic agents in seven studies, bronchodilators and steroids in three studies each, and combined bronchodilator/inhaled corticosteroid in one study.

Fewer adverse events were reported in those who received CHM plus pharmacotherapy (71 events) compared with the pharmacotherapy alone (86 events). For a small number of AEs, the group allocation was not specified (10 events).

Adverse events were analysed according to the nature of the event (see Table 2). The number of gastrointestinal adverse events was greater in CHM plus pharmacotherapy group than pharmacotherapy alone (35 versus 26 events), while the reverse was found for miscellaneous AEs (31 versus 48 , resp.). Nausea was the most frequently reported $\mathrm{AE}$, occurring in 27 events in the CHM plus pharmacotherapy group and 18 events in the pharmacotherapy alone group. Few dermatological events were reported and were similar between groups (10 in CHM plus pharmacotherapy, seven in pharmacotherapy alone). Intravenous injection with Tan re qingcombined with pharmacotherapy was associated with all the dermatological events in the treatment group. A broad range of other AEs were seen. Other frequently reported AEs 


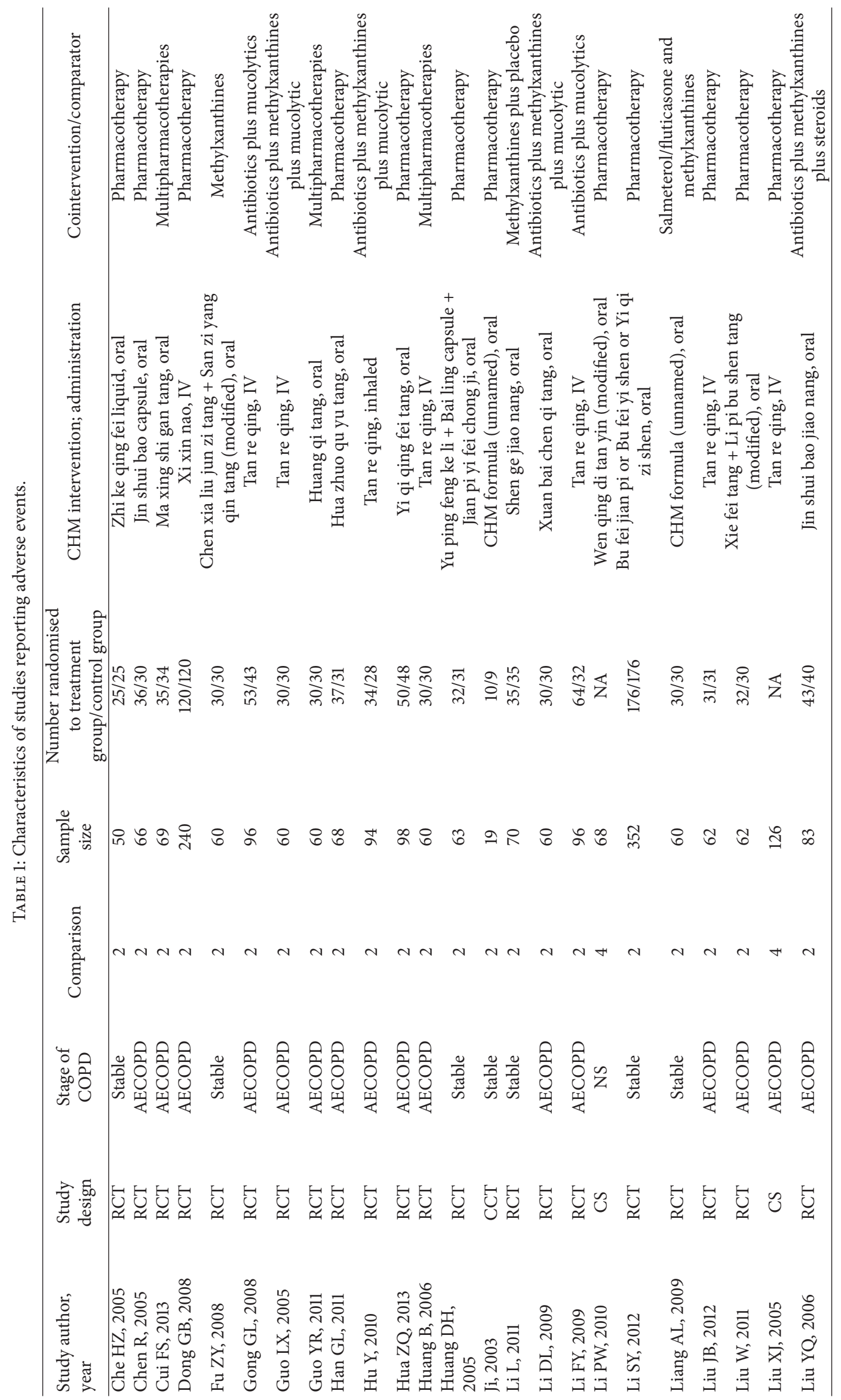




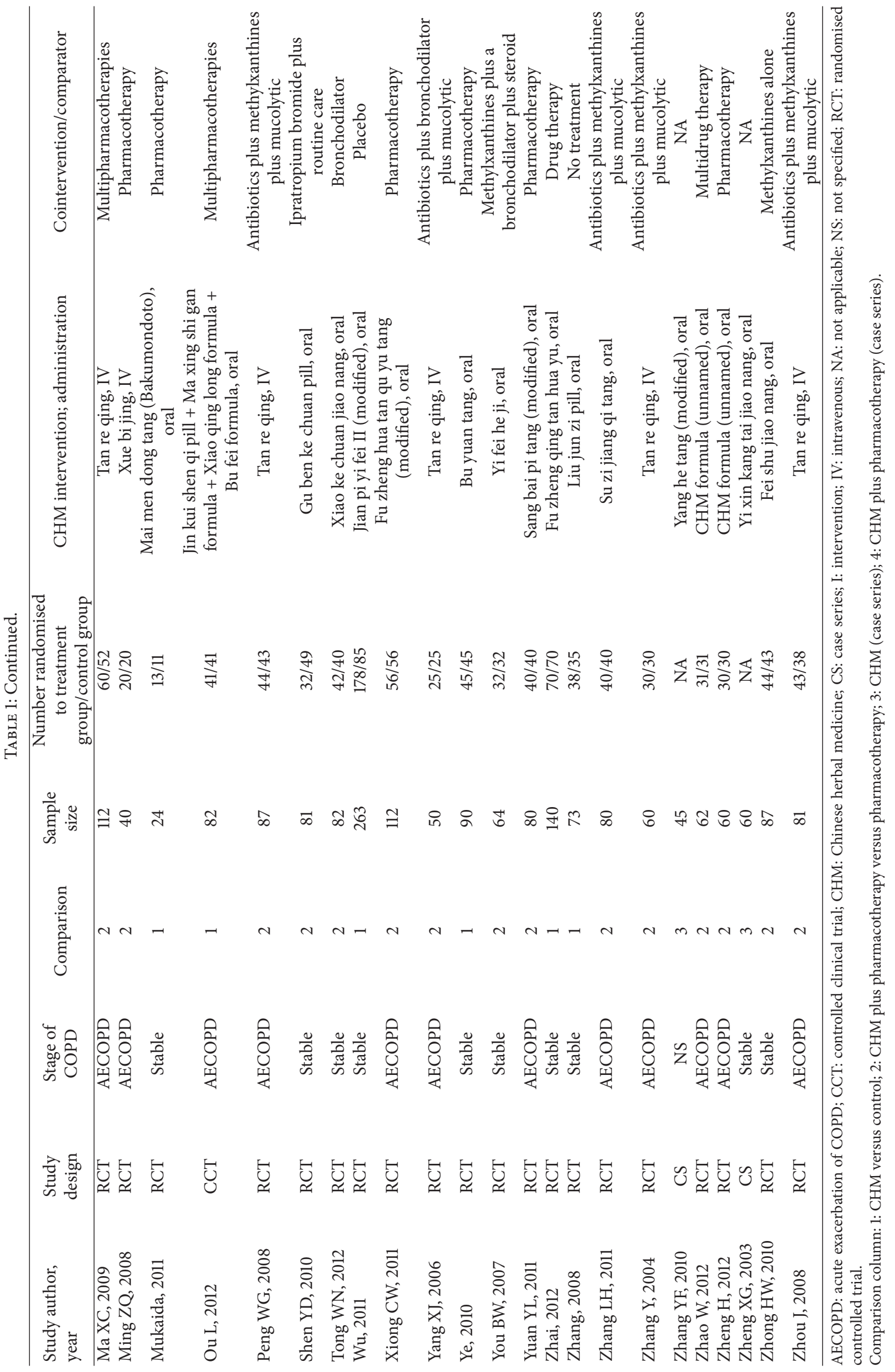


TABLE 2: Summary of adverse events.

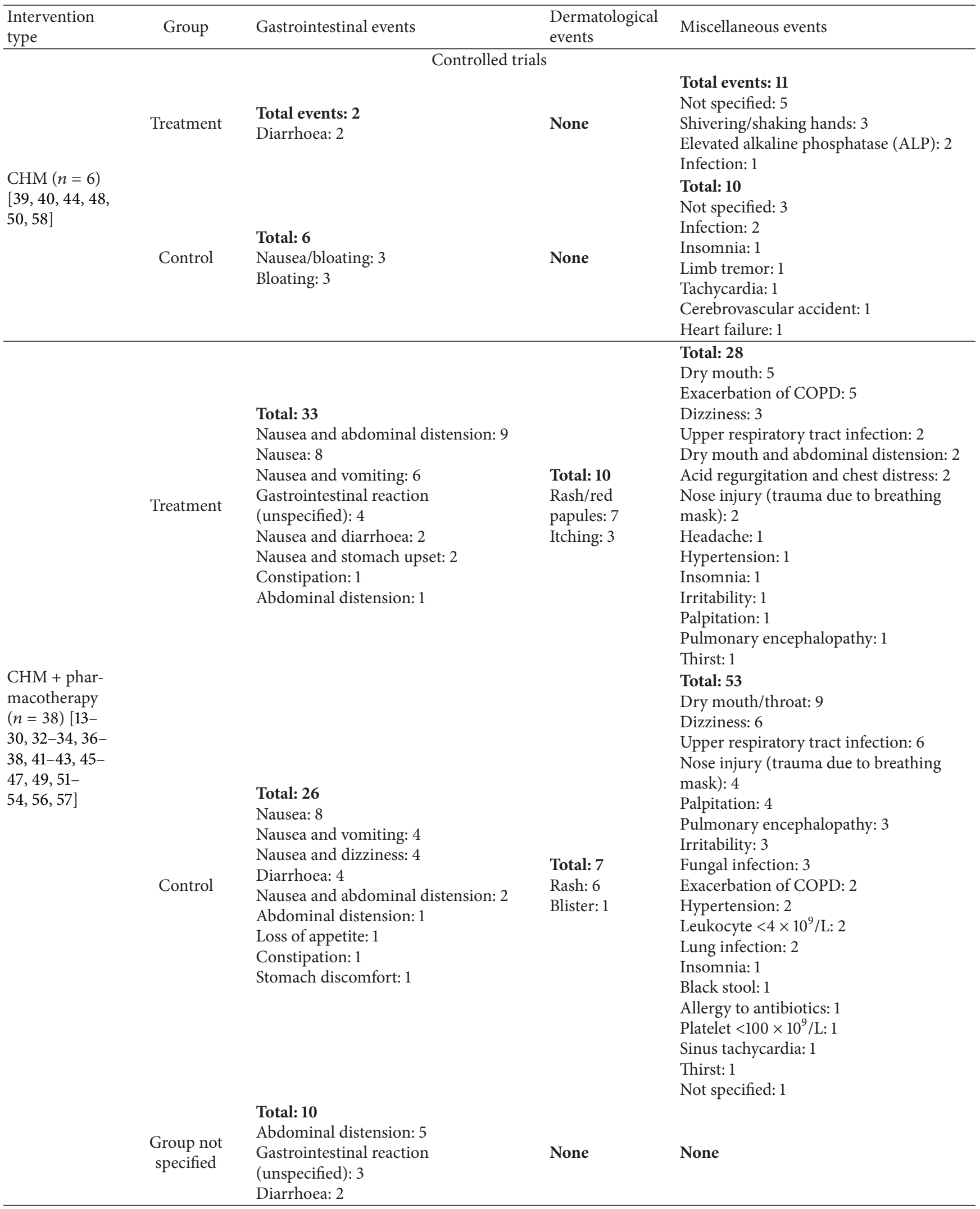


TABLe 2: Continued.

\begin{tabular}{|c|c|c|c|c|}
\hline $\begin{array}{l}\text { Intervention } \\
\text { type }\end{array}$ & Group & Gastrointestinal events & $\begin{array}{l}\text { Dermatological } \\
\text { events }\end{array}$ & Miscellaneous events \\
\hline \multicolumn{5}{|c|}{ Case series } \\
\hline $\begin{array}{l}\mathrm{CHM}(n=2) \\
{[55,59]}\end{array}$ & NA & $\begin{array}{l}\text { Total: } 3 \\
\text { Vomiting: } 2 \\
\text { Nausea: } 1\end{array}$ & None & $\begin{array}{l}\text { Total: } 6 \\
\text { Exacerbation of COPD: } 5 \\
\text { Headache and dizziness: } 1\end{array}$ \\
\hline $\begin{array}{l}\mathrm{CHM}+\text { phar- } \\
\text { macotherapy } \\
(n=2)[31,35]\end{array}$ & NA & $\begin{array}{l}\text { Total: } 1 \\
\text { Abdominal discomfort and nausea: } 1\end{array}$ & None & $\begin{array}{l}\text { Total: } 1 \\
\text { Death (cause unknown): } 1\end{array}$ \\
\hline
\end{tabular}

were dry mouth/throat (5 cases in the treatment groups and 9 cases in the control groups) and dizziness (3 cases in the treatment groups and 6 cases in the control groups).

In studies where the severity of the $\mathrm{AE}$ was reported, all events were described as mild. Sixteen AEs were described as being probably related to $\mathrm{CHM}$ plus pharmacotherapy interventions $[18,21,24,28,33,37,38,41,43,46,52]$, five events were possibly related to treatment [29], and one was unlikely to be related to treatment [22]. Nine reports of AEs in the pharmacotherapy group were considered probably related to treatment $[18,28,37,38,43,46]$ and five possibly related to treatment [29]. Management of AEs was reported in most studies and subsequently all the events resolved. Management included dosage change for $\mathrm{CHM}[15,33,46]$, prescription of other drugs and/or hospital admission [15, 18, 20, 24, 26, $28,41,43,52]$, and no treatment $[20,22]$, and in 11 studies the AE was able to be tolerated whilst continuing treatment $[14,16,23,25,30,36-38,42,54,56]$.

3.2. Noncontrolled Studies. Six case series report on AEs [31, $35,55,59,62,63]$. Adverse events occurred in four studies, two where CHM was used alone $[55,59]$ and two where CHM was combined with pharmacotherapy $[31,35]$. Nine AEs were reported in those who received CHM alone, and two events occurred where CHM was combined with pharmacotherapy. Exacerbation of COPD was the most common AE in the noncontrolled studies (5 events). Exacerbations were managed with antibiotics [55]. Other AEs included vomiting (2 events) [59], and one case each included nausea [59], headache and dizziness [59], abdominal discomfort and nausea [35], and patient death (reason not given) [31]. None of the studies reported the severity of the AE or assigned causality. The one participant who reported abdominal discomfort and nausea continued treatment [35], and the headache and dizziness in one participant resolved without intervention [59].

\section{Discussion}

Overall, the rate of AEs in clinical studies of CHM for COPD was low $(1.7 \%)$. The rate of AEs in participants who received CHM intervention was slightly lower than control groups, $1.4 \%$ versus $1.8 \%$. This result fell well within the range of minimal risk for minor complications $(0.1 \%$ to $10 \%)$ suggested by MacPherson et al. [64]. Where described, the majority of AEs were mild in severity. CHM appears to be well tolerated by patients with COPD.

Nausea was the most common AE in both the treatment groups and control groups but was higher amongst those who received CHM. The majority of nausea AEs occurred in people with an acute exacerbation of COPD. It is difficult to determine whether this is related to exacerbation, the CHMs, or the pharmacotherapy administered concurrently. Whilst the number of AEs which included nausea was higher in those who received CHM, this was within the range for minimal risk [64] and overall the data shows that CHM in combination with pharmacotherapy does not pose any additional risks for people with acute exacerbations of COPD. The data shows no clusters of particular events. A causal relationship to treatments was not evident and the AEs may reflect general fluctuations in the patients' conditions.

Many of the pharmacotherapies used in included studies have established safety profiles and known AEs. For example, bronchodilators can cause tremor, tachycardia, headaches, nausea, palpitations, hypokalaemia, and dry mouth, and corticosteroids can cause pneumonia, oral candidiasis, and skin bruising [65]. Among studies of CHM plus pharmacotherapy versus pharmacotherapy alone the number of AEs was lower amongst those who received CHM. In clinical practice, the choice of CHM can be influenced by the known safety profile of particular pharmacotherapies, with herbs added to address known side effects. The finding from this review suggests that $\mathrm{CHM}$ may reduce the number of AEs in people with COPD, although further research is needed to confirm this finding as the differences seen were small.

People with COPD often have comorbidities requiring polydrug therapy, which may increase the potential for herbdrug interactions. Indeed, there is significant potential for adverse drug reactions in elderly people with complex and chronic health conditions such as COPD and asthma [66]. Elderly people with COPD who are taking CHM in addition to prescribed medications should be monitored for the emergence of new signs or symptoms and should be reviewed regularly.

The data presented in this review is important because there are no existing reviews of clinical studies on the safety of CHM for COPD. While population and practitioner surveys may be more reflective of clinical practice, AEs reported in population surveys may differ from those in clinical 
TABLE 3: CHM formula and ingredients of studies with adverse events in treatment groups.

\begin{tabular}{|c|c|c|}
\hline CHM intervention; route of administration & Herbal ingredients & References \\
\hline $\begin{array}{l}\text { Bu fei jian pi or Bu fei yi shen or Yi qi zi shen, } \\
\text { oral }\end{array}$ & $\begin{array}{l}\text { Bu fei jian pi: huang qi, dang shen, bai zhu, fu ling, and } \\
\text { chuan bei mu; Bu fei yi shen: ren shen, huang qi, gou qi zi, } \\
\text { shan zhu yu, and yin yang huo; Yi qi zi shen: ren shen, } \\
\text { huang jing, shu di huang, mai dong, and wu wei zi }\end{array}$ & {$[29]$} \\
\hline $\begin{array}{l}\text { Chen xia liu jun zi tang + San zi yang qin tang } \\
\text { (modified), oral }\end{array}$ & $\begin{array}{c}\text { Dang shen, bai zhu, fu ling, chen pi, fa xia, su zi, lai fu zi, } \\
\text { bai jie zi, tao ren, zhe bei mu, bei xing, hai ge qiao, and gan } \\
\text { cao }\end{array}$ & {$[17]$} \\
\hline CHM formula (unnamed), oral & $\begin{array}{l}\text { Ma huang, bai guo, sang bai pi, zhe bei mu, gua lou, huang } \\
\text { qin, su zi, zi he che, and ge jie }\end{array}$ & {$[54]$} \\
\hline CHM formula (unnamed), oral & $\begin{array}{l}\text { Fa ban xia, fu ling, chuan xiong, chao gu ya, chao mai ya, } \\
\text { bai he, shan yao, sha shen, fo shou, shan zha, jin fei cao, } \\
\text { and zhe bei mu }\end{array}$ & {$[26]$} \\
\hline Fei shu jiao nang, oral & $\begin{array}{l}\text { Hong shen, ge jie, san qi, chuan bei mu, di long, chuan } \\
\text { xiong, and gan cao }\end{array}$ & {$[56]$} \\
\hline Fu zheng hua tan qu yu tang (modified), oral & Zhi fu zi, bu gu zi, ting li zi, chuan xiong, and yu jin & {$[45]$} \\
\hline Fu zheng qing tan hua yu, oral & $\begin{array}{l}\text { Sheng shai shen/ren shen, ha jie, wu wei zi, xing ren, gua } \\
\text { lou, xie bai, dan shen, tao ren, and mao dong qing }\end{array}$ & {$[50]$} \\
\hline Gu ben ke chuan pill, oral & NS & {$[42]$} \\
\hline Hua zhuo qu yu tang, oral & $\begin{array}{c}\text { Chi shao, dan shen, tao ren, hong hua, gua lou, su mu, zi } \\
\text { su zi, zhe bei mu, fu ling, kuan dong hua, yu xing cao, and } \\
\text { gan cao }\end{array}$ & {$[21]$} \\
\hline Huang qi tang, oral & $\begin{array}{l}\text { Huang qi, dang shen, bai zhu, gua lou, xing ren, tao ren, } \\
\text { chuan xiong, dang gui, chen pi, and huang qin }\end{array}$ & {$[20]$} \\
\hline Jian pi yi fei II (modified), oral & Dang shen, bai zhu, suo yang, and tao ren & {$[44]$} \\
\hline
\end{tabular}

Jin kui shen qi pill: shu di huang, shan zhu yu, shan yao,

shu fu zi, gui zhi, bu gu zhi, fu ling, tao ren, ze xie, and wu wei zi

Ma xing shi gan formula: ma huang, xing ren, huang qin, jie geng, sheng shi gao, yu xing cao, jin yin hua, lian qiao, zhe bei, tao ren, and gan cao

Jin kui shen qi pill + Ma xing shi gan formula + Xiao qing long formula $+\mathrm{Bu}$ fei formula, oral

Xiao qing long formula: gui zhi, bai shao, gan jiang, ma

huang, ban xia, zhe bei, xi xin, chuan xiong, wu wei zi, and gan cao

Bu fei formula: shu di, huang qi, dang shen, sang bai pi, zi

yuan, kuan dong hua, qian hu, chen pi, wu wei zi, dan shen, su zi, and bai jie zi

Jin shui bao jiao nang, oral Jin shui bao jiao nang

Liu jun zi pill, oral NS

Ma xing shi gan tang, oral

Ma huang, xing ren, shi gao, gan cao, lai fu zi, ting li zi, fu ling, jv hong, zhu ru, shi chang pu, yu jin, gui zhi, fu ling, bai zhu, zhu ling, and ze xie

Mai men dong tang (Bakumondoto), oral

Mai men dong, ban xia, gu ya, da zao, ren shen, and gan cao

\begin{tabular}{l}
\hline Shen ge jiao nang, oral \\
Tan re qing, inhaled
\end{tabular}

Ren shen, chuan bei, zi he che, and zhi ge jie NS

Tan re qing, IV

Huang qin, jin yin hua, lian qiao, xiong dan fen, and shan

$$
\text { yang jiao }
$$

$[18,19,24,28$,

$33,35,37,41$, $46,52]$

Wen qing di tan yin (modified), oral

Jiang ban xia, bei xi xin, wu wei zi, gan jiang, ting li zi, fu

ling, and wang jiang nan. If yin deficiency, add sha shen, mai dong; if phlegm heat, add sang bai pi, gua lou pi

Xi xin nao, IV NS

Xiao ke chuan jiao nang, oral

Man shan hong

[43]

Xue bi jing, IV NS 
TABLE 3: Continued.

\begin{tabular}{lc}
\hline CHM intervention; route of administration & Herbal ingredients \\
\hline Yang he tang (modified), oral & $\begin{array}{c}\text { Su di, su zi, rou gui, gan cao, zhi ma huang, lu jiao jiao, bai } \\
\text { jie zi, fa xia, shu mu, chen pi, and pao jian. If with cough, } \\
\text { add chuan bei, zhi zhi wan; if with phlegm, add gua lou } \\
\text { ren, cao miao zi; if spleen qi deficiency, add dang shen, bai } \\
\text { zhu; if blood stasis, add dan shen, tao ren, yi mu cao; if } \\
\text { with heat, remove rou gui, lu jiao jiao, and su di and add } \\
\text { sang bai pi, zhe bei, se gan, and huang qin }\end{array}$ \\
\hline Yi qi qing fei tang, oral & $\begin{array}{c}\text { Huang qi, dan shen, huang qin, bei mu, ban xia, jie geng, } \\
\text { gua lou qiao, and yu xing cao }\end{array}$ \\
\hline Yi xin kang tai jiao nang, oral & $\begin{array}{c}\text { Tang gu te qian xian lian, duo xian xian gou zi, tang gu te } \\
\text { da huang, and shuo yang }\end{array}$ \\
\hline $\begin{array}{l}\text { Yu ping feng ke li + Bai ling capsule + Jian pi yi } \\
\text { fei chong ji, oral }\end{array}$ & $\begin{array}{c}\text { Yu ping feng ke li: NS; Bai ling capsules: NS; Jian pi yi fei } \\
\text { chong ji: ren shen, bai zhu, fu ling, mai dong, sang bai pi, } \\
\text { and huang qi }\end{array}$ \\
\hline
\end{tabular}

IV: intravenous; NS: not specified.

studies because of known controllable risks associated with CHM. These include misidentification of herbal medicines, substitution, lack of standardisation, contamination, and adulteration, as well as some herbal ingredients not being listed on the product labels $[67,68]$. In the clinical trial setting, the herbal medicine(s) is more likely to be controlled for these issues and any subsequent AEs can be better understood in the context of specific causality rather than poor manufacturing or handling.

The challenges of conducting systematic reviews of CHM have been described by White [69]. This review found a lower rate of AEs than that of MacPherson and Liu [6]. Few systematic reviews have evaluated the safety of CHM, and none have focused on patients with COPD. This may in part be due to the many and varied herbal formulae used in clinical trials and variation in publication keywords which limits electronic database searching. Further, the incidence of AEs is challenging to calculate as it requires adequate reporting of AEs in clinical studies (e.g., number and nature of events and authors definition of AE), information on the number of consultations, and whether the CHM was changed during the course of treatment. Additionally, some studies only provided limited and vague statements about AEs, such as "there were no AEs" or "CHM was safe." Therefore the actual frequency of AEs may be underestimated.

Reporting of details of AEs in studies included in this review was limited largely to the nature and number of AEs, with scant details on severity, causality, management, and outcome. As AE data can be influenced by the manner in which it is collected [70], future clinical studies should describe the process for collection of safety data. Further, AE data in future clinical studies of CHM for COPD should be reported in accordance with the CONSORT extension for reporting of harms in randomised trials [71].

Finally, the diversity of CHM formulae and individual herbs used in included studies did not allow for greater exploration of AEs by individual formula (with the exception of Tan re qing injection) or by individual herb. In CHM formula with multiple herb ingredients, it is difficult to attribute AEs to one herb or the synergistic actions of all herb ingredients. Thus, the findings of this review are limited to describing the potential for an $\mathrm{AE}$ when any $\mathrm{CHM}$ formula is used. The selection of CHM formula for people with COPD should consider relevant available safety data and the concomitant medications being used by the patient.

\section{Conclusion}

Overall, CHM is well tolerated by patients with COPD. The rate of AEs is low, minor, and self-resolving and was slightly less than that of pharmacotherapy, placebo, and no treatment. The most common AE was nausea, which was seen when CHM was combined with pharmacotherapy for acute exacerbations of COPD. In addition, CHM appeared to be safe when taken in combination with pharmacotherapies. Future research should provide greater details relating to AEs in order to more accurately inform clinical practice.

\section{Conflict of Interests}

The authors declare that there is no conflict of interests regarding the publication of this paper.

\section{Acknowledgments}

The authors would like to acknowledge the contributions of Dr. Xuhua Yu and Dr. Yuan Ming Di who assisted with data extraction and verification and Professor Lin Lin who provided clinical advice. The project is jointly supported by the China-Australia International Research Centre for Chinese Medicine (CAIRCCM), a joint initiative of RMIT University, Australia, and the Guangdong Provincial Academy of Chinese Medical Sciences, China, with additional funding support from the Ministry of Science \& Technology of China (International Cooperation Project, Grant no. 02012DFA31760) and the National Health and Medical Research Council (NHMRC), project Grant no. 616609. 


\section{References}

[1] J. George, L. L. Ioannides-Demos, N. M. Santamaria, D. C. M. Kong, and K. Stewart, "Use of complementary and alternative medicines by patients with chronic obstructive pulmonary disease," Medical Journal of Australia, vol. 181, no. 5, pp. 248251, 2004.

[2] O. Abadoglu, E. Cakmak, and S. K. Demir, "The view of patients with asthma or chronic obstructive pulmonary disease (COPD) on complementary and alternative medicine," Allergologia et Immunopathologia (Madr), vol. 36, no. 1, pp. 21-25, 2008.

[3] T. Y. K. Chan and J. A. J. H. Critchley, "Usage and adverse effects of Chinese herbal medicines," Human and Experimental Toxicology, vol. 15, no. 1, pp. 5-12, 1996.

[4] X. An, A. L. Zhang, A. W. Yang et al., "Oral ginseng formulae for stable chronic obstructive pulmonary disease: a systematic review," Respiratory Medicine, vol. 105, no. 2, pp. 165-176, 2011.

[5] X. An, A. L. Zhang, B. H. May, L. Lin, Y. Xu, and C. C. Xue, "Oral Chinese herbal medicine improvement of quality of life for stable chronic obstructive pulmonary disease: a systematic review," The Journal of Alternative and Complementary Medicine, vol. 18, no. 8, pp. 731-743, 2012.

[6] H. MacPherson and B. Liu, "The safety of Chinese herbal medicine: a pilot study for a national survey," Journal of Alternative and Complementary Medicine, vol. 11, no. 4, pp. 617626, 2005.

[7] T. Y. K. Chan, A. Y. W. Chan, and J. A. J. H. Critchley, "Hospital admissions due to adverse reactions to Chinese herbal medicines," Journal of Tropical Medicine and Hygiene, vol. 95, no. 4, pp. 296-298, 1992.

[8] J. T. Coon and E. Ernst, "Panax ginseng: a systematic review of adverse effects and drug interactions," Drug Safety, vol. 25, no. 5, pp. 323-344, 2002.

[9] E. Ernst, "Adverse effects of herbal drugs in dermatology," British Journal of Dermatology, vol. 143, no. 5, pp. 923-929, 2000.

[10] R. Guo, M. H. Pittler, and E. Ernst, "Herbal medicines for the treatmet of COPD: a systematic review," European Respiratory Journal, vol. 28, no. 2, pp. 330-338, 2006.

[11] A. Bensoussan, S. P. Myers, and A.-L. Carlton, "Risks associated with the practice of traditional Chinese medicine: an Australian study," Archives of Family Medicine, vol. 9, no. 10, pp. 1071-1078, 2000.

[12] Cochrane Airways Group, Developing a Search Strategy for an Airways Group Systematic Review, 2012, http://airways.cochrane.org/sites/airways.cochrane.org/files/uploads/ Search\%20methods\%20guide\%20V1.3.pdf.

[13] H. Che, J. Pang, C. Li, and Y. Wang, "Effects of Zhike Qingfei oral liquid on inflammatory cells, interleukin-8 and tumour necrosis factor-a in the sputum in 25 cases of chronic obstructive pulmonary diseases," Journal of Traditional Chinese Medicine, vol. 46, no. 10, pp. 759-761, 2005.

[14] H. Che, J. Pang, and C. Li, "Effects of Zhike Qingfei oral liquid on inflammatory cells, interleukin- 8 and tumour necrosis factor-a in the sputum in 25 cases of chronic obstructive pulmonary diseases," Journal of Traditional Chinese Medicine, vol. 46, no. 10, pp. 759-761, 2005.

[15] F. Cui, "Modified Ma Xing Shi Gan Tang in combination with continuous positive airway pressure for acute exacerbation of chronic pulmonary disease, 35 cases," Henan Traditional Chinese Medicine, vol. 33, no. 2, pp. 183-184, 2013.

[16] G. Dong, Z. Zhang, C. Li, and C. Han, "Clinical observation of 120 cases of Xi Xin Nao injections for chronic pulmonary disease," Journal of Binzhou Medical University, vol. 31, no. 2, pp. 141-142, 2008.

[17] Z. Fu, The Effect of Tonify Spleen, Remove Phlegm and Invigorate Blood Method on the Lung Function and Qualify of Life for Stable Stage of Chronic Pulmonary Disease, Guangzhou University of Chinese Medicine, 2008.

[18] G. Gong, "Combined therapy of Chinese medicine and western medicine for the treatment of COPD in the elderly," Modern Journal of Integrated Traditional Chinese and Western Medicine, vol. 17, no. 36, pp. 5595-5596, 2008.

[19] L. Guo and X. Xu, "Clinical observation of the effect of Tan re qing injections for AECOPD treatment," Journal of Clinical Pulmonary Medicine, vol. 10, no. 6, pp. 803-804, 2005.

[20] Y. Guo, The Clinical Observation of Tonify Qi and Invigorate Blood Method for the Treatment of AECOPD Patients with Qi Deficiency and Blood Stasis, Shandong University of Traditional Chinese Medicine, 2011.

[21] G. Han and S. Zhang, "The effect of removing turbidity and stasis method in the treatment of acute exacerbation of chronic pulmonary disease," Beijing University of Traditional Chinese Medicine, vol. 30, no. 2, pp. 94-96, 2011.

[22] Y. Hu and C. Yang, "Combined therapy of Chinese medicine and Western medicine treatment in acute exacerbation of chronic pulmonary disease," Journal of Practical Traditional Chinese Medicine, vol. 26, no. 10, pp. 698-699, 2010.

[23] Z.-Q. Hua and Y.-J. Chang, "Clinical study of decoction of invigorating Qi and clearing lung combined standardized myrtol on acute exacerbation of chronic obstructive pulmonary disease (AECOPD)," China Journal of Chinese Materia Medica, vol. 38, no. 3, pp. 440-442, 2013.

[24] B. Huang, Q. Huang, D. Lu, and C. Xie, "Clinical study of Tanreqing injections for acute exacerbation in chronic pulmonary disease," Journal of Emergency in Traditional Chinese Medicine, vol. 15, no. 5, pp. 464-466, 2006.

[25] D. H. Huang, L. Wu, D. He, and L. Lin, "Clinical observation of tranquilization period of chronic obstructive pulmonary disease treated by integration of traditional Chinese medicine and Western medicine," Journal of the Fourth Military Medical University, vol. 26, no. 17, pp. 1611-1613, 2005.

[26] H. Ji, G. Hu, J. Gao, and S. Wang, "Clinical observation of Tonify Qi and Yin method for stable chronic pulmonary disease patients with malnutrition," Shandong Journal of Chinese Medicine, vol. 22, no. 1, pp. 11-12, 2003.

[27] D. Li, Clinical Study of Modified Xuan Bai Cheng Qi Tang for Chronic Pulmonary Disease, Liaoning University of Chinese Medicine, 2009.

[28] F. Li, "The treatment effect of combined Chinese medicine and Western medicine for acute exacerbation of chronic pulmonary disease in the elderly," Chinese Health Care, vol. 17, no. 18, pp. 716-717, 2009.

[29] S.-Y. Li, J.-S. Li, M.-H. Wang et al., "Effects of comprehensive therapy based on traditional Chinese medicine patterns in stable chronic obstructive pulmonary disease: a four-center, openlabel, randomized, controlled study," BMC Complementary and Alternative Medicine, vol. 12, article 197, 2012.

[30] L. Li, S. Zhang, and L. Shu, "35 cases of Shenha capsules for the treatment of COPD patients with Lung and Kidney deficiency plus phlegm obstruction," Guiding Journal of Traditional Chinese Medicine and Pharmacy, vol. 17, no. 12, pp. 45-46, 2011.

[31] P. Li, "The effects of combined therapy of Chinese and Western medicine for the treatment of COPD, 68 cases," Guang Ming Chinese Medicine, vol. 25, no. 5, p. 845, 2010. 
[32] A. Liang, The Effect of Tonify Lung, Spleen and Kidney Method on Patients Quality of Life in Chronic Pulmonary Disease, Beijing University of Chinese Medicine, 2009.

[33] J. Liu, "Observation of the therapeutic effect of Tanreqing injection on obstructive pulmonary emphysema," Pharmacology and Clinics of Chinese Materia Medica, vol. 28, no. 2, pp. 170-172, 2012.

[34] W. Liu, "Combine therapy using syndrome differentiation and western medicine for the treatment of servere COPD, 32 cases," Shanxi Journal of Chinese Medicine, vol. 32, no. 6, pp. 702-704, 2011.

[35] X. Liu, M. Tu, W. Liu, H. Lei, and G. Luo, "The effect of Tanreqing injections for acute exacerbation of COPD," Clinical Medicine, vol. 25, no. 9, pp. 60-61, 2005.

[36] Y. Liu, "Combined therapy of Jin Shui Bao capsules and Western medicine for the treatment of acute exacerbation of chronic pulmonary disease, 43 cases," Journal of Practical Traditional Chinese Medicine, vol. 22, no. 4, pp. 224-225, 2006.

[37] X. Ma and H. Li, "A study of tanreqing plus levofloxacin fot the treatment of acute exacerbation of chronic pulmonary disease," Guide of China Medicine, vol. 7, no. 18, pp. 69-70, 2009.

[38] Z. Ming, L. Yu, L. Wang, and Y. Lv, "Effects of Xuebing injection on the clinical efficacy of noninvasive ventilation in patients with chronic obstructive pulmonary disease," Journal of Emergency in Traditional Chinese Medicine, vol. 17, no. 8, pp. 1047-1049, 2008.

[39] K. Mukaida, N. Hattori, K. Kondo et al., "A pilot study of the multiherb Kampo medicine bakumondoto for cough in patients with chronic obstructive pulmonary disease," Phytomedicine, vol. 18, no. 8-9, pp. 625-629, 2011.

[40] L. Ou and J. Huang, "Analyze the effect of Chinese and Western medicine combination for acute attack of chronic obstructive pulmonary emphysema," The Journal of Medical Theory and Practice, vol. 25, no. 15, pp. 1817-1818, 2012.

[41] W. Peng, "Clinical observation of Tan Re Qing injection for the treatment of acute exacerbation of COPD, 87 cases," Asia Pacific Traditional Medicine, vol. 4, no. 11, pp. 67-68, 2008.

[42] Y. Shen, "Clinical observation of inhaled ipratropium bromide plus $\mathrm{Gu}$ ben ke chuan pills for the treatment of chronic obstructive pulmonary disease," China Modern Medicine, vol. 17, no. 34, pp. 63-65, 2010.

[43] W. Tong, Y. Cao, and A. Zhuo, "The clinical observation of Xiao ke chuan capsules plus tiotropium bromide for the treatment of stable chronic obstructive pulmonary disease (aboe stage 3 ) in the elderly," Chinese Journal of Information on Traditional Chinese Medicine, vol. 19, no. 3, pp. 79-80, 2012.

[44] L. Wu, L. Lin, Y. Xu et al., "Clinical research on 178 cases of chronic obstructive pulmonary disease in the stable stage treated with Jianpi Yifei II," Journal of Traditional Chinese Medicine, vol. 52, no. 17, pp. 1465-1468, 2011.

[45] C. Xiong, M. Zou, J. Zhang, and H. Xiao, "Integrated traditional and western treatment of chronic obstructive pulmonary disease with acute exacerbation of the effect," Chinese and Foreign Medical Research, vol. 9, no. 24, pp. 11-12, 2011.

[46] X. Yang, "The effect of Tanreqing injection in the treatment of acute exacerbation of chronic obstructive pulmonary disease," Health Horizon Medical Science Fascicule, vol. 2, p. 82, 2006.

[47] Y. Yuan, Y. Deng, and Y. Hu, "Clinical observation of Sang bai pi tang for the treatment of AECOPD patients with Phlegm heat obstructing the Lung syndrome," Journal of Sichuan of Traditional Chinese Medicine, vol. 29, no. 11, p. 81, 2011.
[48] C. Ye, H. Xue, B. Fu et al., "The clinical study of Bu-yuan decoction interfering in the chronic obsttructive pulmonary disease in stable phase," Journal of Jiangxi University of TCM, vol. 22, no. 1, pp. 54-57, 2010.

[49] B. You, Clinical Research of the Effect of Yi Fei He Ji on the Quality of Life for COPD Patients, Hunan University of Traditional Chinese Medicine, 2007.

[50] R. Zhai and H. Zhai, "Clinical study of Fu Zheng Qing Tan Hua Yu capsules for the treatment of stable chronic obstructive pulmonary disease," Journal of Practical Traditional Chinese Medicine, vol. 28, no. 11, pp. 900-902, 2012.

[51] L. Zhang and J. Wu, "Effect of Suzi Jiangqi decoction combined with Western medicine on acute exacerbation of chronic obstructive pulmonary disease," Chinese Medicine Modern Distance Education of China, vol. 9, no. 15, pp. 48-49, 2011.

[52] Y. Zhang, T. Li, G. Wang et al., "Randomized controlled trial of TanReqing injection in treatment of acute exacerbation of chronic obstructive pulmonary disease (syndrome of retention of phlegm-heat in the lung)," Chinese Journal of Evidence-Based Medicine, vol. 4, no. 5, pp. 300-305, 2004.

[53] W. Zhao and Z. Liu, "The efficacy of integrated traditional Chinese medicine and western medicine for treatment of patients with acute exacerbation of chronic obstructive pulmonary disease," Chinese Journal of Integrated Traditional and Western Medicine in Intensive and Critical Care, vol. 19, no. 4, pp. 232234, 2012.

[54] H. Zheng, Clinical observation of Qingfei Bushen method on treating chronic obstructive pulmonary disease in acute exacerbation [M.S. thesis], Shandong University of Traditional Chinese Medicine, 2012.

[55] X. Zheng, C. Chen, Q. Chen, and Y. Wang, "Clinical observation of Yi Xin Kang Tai capsules for chronic obstructive pulmonary disease," Practical Clinical Journal of Integrated Traditional Chinese and Western Medicine, vol. 3, no. 3, p. 15, 2003.

[56] H. Zhong, S. Peng, Y. Luo, H. Gao, S. Ao, and J. Wang, "The clinical effect of Feishu capsule on steady stage of COPD," Tianjin Medical Journal, vol. 38, no. 10, pp. 849-851, 2010.

[57] J. Zhou, B. Wu, and F. Cai, "The effect of Tanreqing plus Lomefloxacin in the treatment of acute exacerbation of chronic pulmonary disease in the elderly," China Foreign Medical Treatment, vol. 23, p. 73, 2008.

[58] X. Zhang and D. Cheng, "The impact of Liu jun zi wan (pills) for BODE index in stable COPD," Yunnan Journal of Traditional Chinese Medicine and Materia Medica, vol. 29, no. 8, pp. 8-9, 2008.

[59] Y. Zhang, "Clinical observation of modified Yang he tang (decoction) for COPD," Chinese Journal of Modern Drug Application, vol. 4, no. 5, pp. 123-124, 2010.

[60] D. Gross, Z. Shenkman, B. Bleiberg, M. Dayan, M. Gittelson, and R. Efrat, "Ginseng improves pulmonary functions and exercise capacity in patients with COPD," Monaldi Archives for Chest Disease-Pulmonary Series, vol. 57, no. 5-6, pp. 242-246, 2002.

[61] X. Zhang, T. Yin, X. Yang, H. Wu, and J. Yao, "Effect of Lifei Yishen-recipe on lung function of COPD Grade III," Chinese Journal of Experimental Traditional Medical Formulae, vol. 19, no. 2, pp. 289-290, 2013.

[62] W. Xue, "Observation of combined therapy of Chinese medicine and Western medicine for chronic obstructive pulmonary disease," Guang Ming Chinese Medicine, vol. 27, no. 8, pp. 16221623, 2012. 
[63] S. Liu, "Acute exacerbations of chronic obstructive pulmonary disease treated by integrated traditional Chinese and western medicine," Clinical Medical Engineering, vol. 18, no. 3, pp. 371372, 2011.

[64] H. MacPherson, A. White, and A. Bensoussan, "The safety of acupuncture," in Acupuncture Research: Strategies for Establishing an Evidence Base, H. MacPherson, Ed., pp. 57-76, Churchill Livingstone, Philadelphia, Pa, USA, 2008.

[65] Global Initiative for Chronic Obstructive Lung Disease, Global Strategy for the Diagnosis, Management, and Prevention of Chronic Obstructive Pulmonary Disease, Global Initiative for Chronic Obstructive Lung Disease, 2013.

[66] D. M. Newnham, "Asthma medications and their potential adverse effects in the elderly: recommendations for prescribing," Drug Safety, vol. 24, no. 14, pp. 1065-1080, 2001.

[67] G. Pinn, "Adverse effects associated with herbal medicine," Australian Family Physician, vol. 30, no. 11, pp. 1070-1075, 2001.

[68] C. W. Cheng, Z. X. Bian, Y. P. Li et al., “Transparently reporting adverse effects of traditional Chinese medicine interventions in randomized controlled trials," Journal of Chinese Integrative Medicine, vol. 6, no. 9, pp. 881-886, 2008.

[69] A. White, "A cumulative review of the range and incidence of significant adverse events associated with acupuncture," Acupuncture in Medicine, vol. 22, no. 3, pp. 122-133, 2004.

[70] Y. K. Loke, D. Price, and A. Herxheimer, "Systematic reviews of adverse effects: framework for a structured approach," $B M C$ Medical Research Methodology, vol. 7, article 32, 2007.

[71] J. P. A. Ioannidis, S. J. W. Evans, P. C. Gøtzsche et al., "Better reporting of harms in randomized trials: an extension of the CONSORT statement," Annals of Internal Medicine, vol. 141, no. 10, pp. 781-788, 2004. 


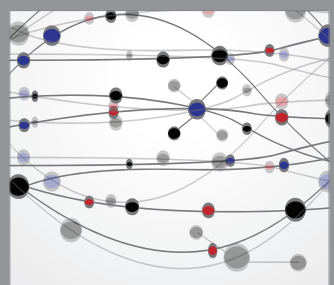

The Scientific World Journal
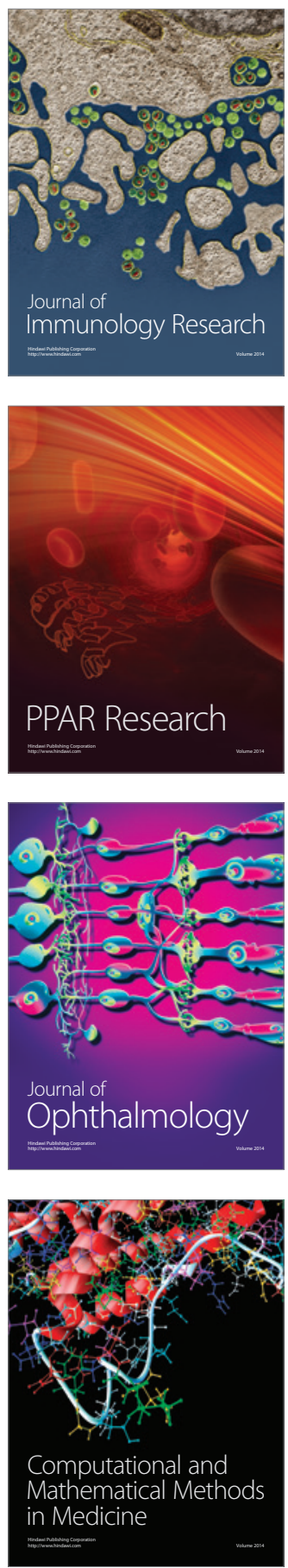

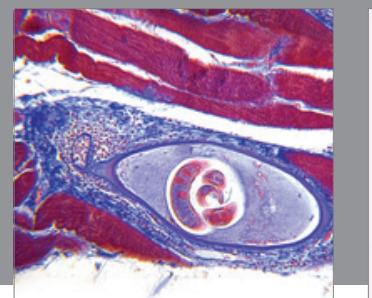

Gastroenterology

Research and Practice
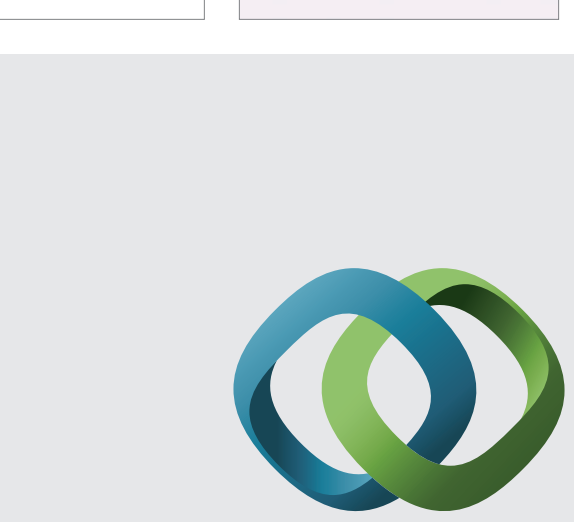

\section{Hindawi}

Submit your manuscripts at

http://www.hindawi.com
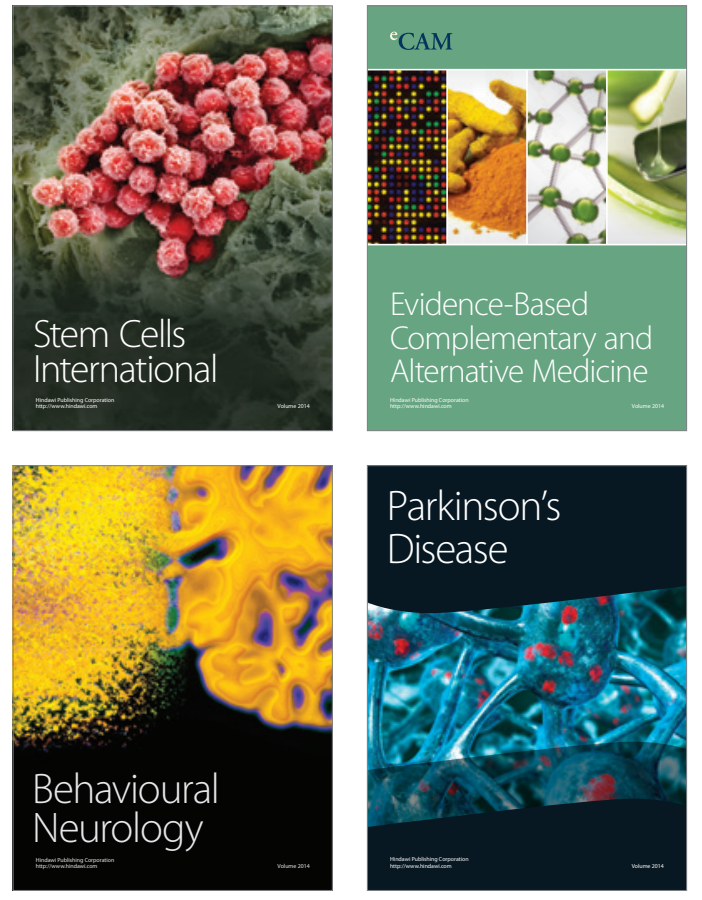
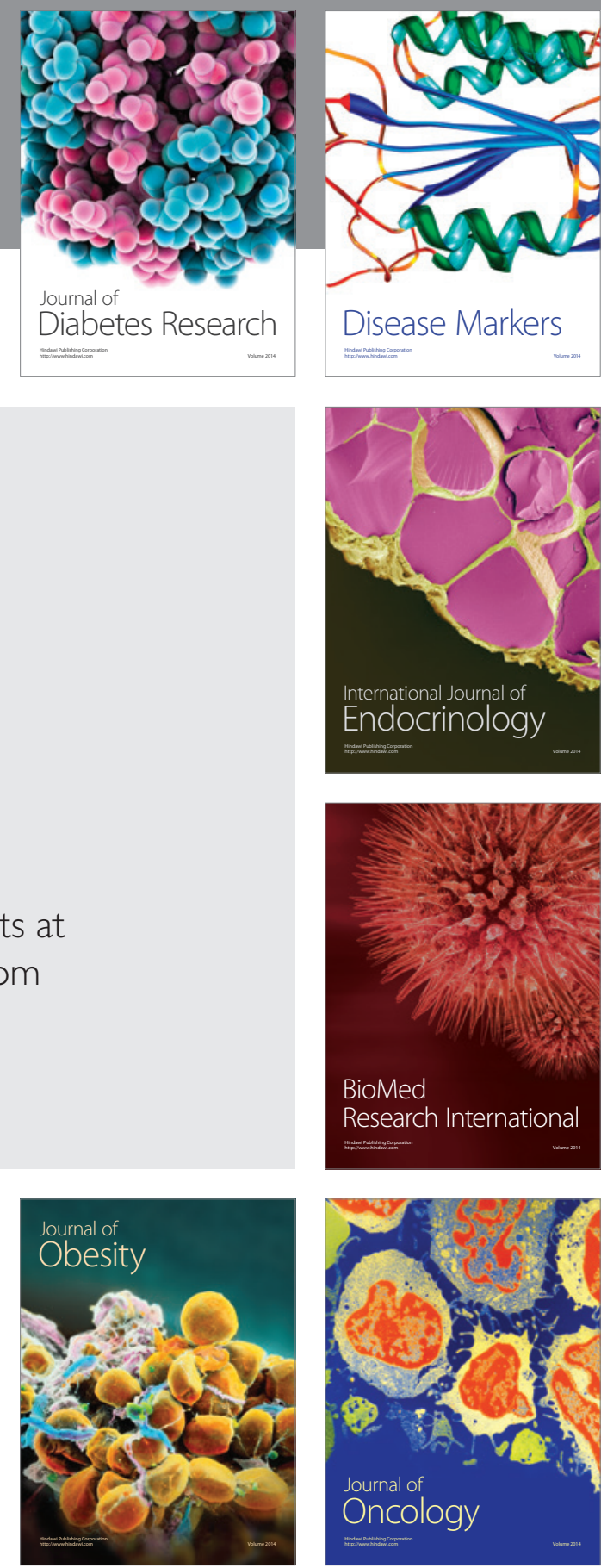

Disease Markers
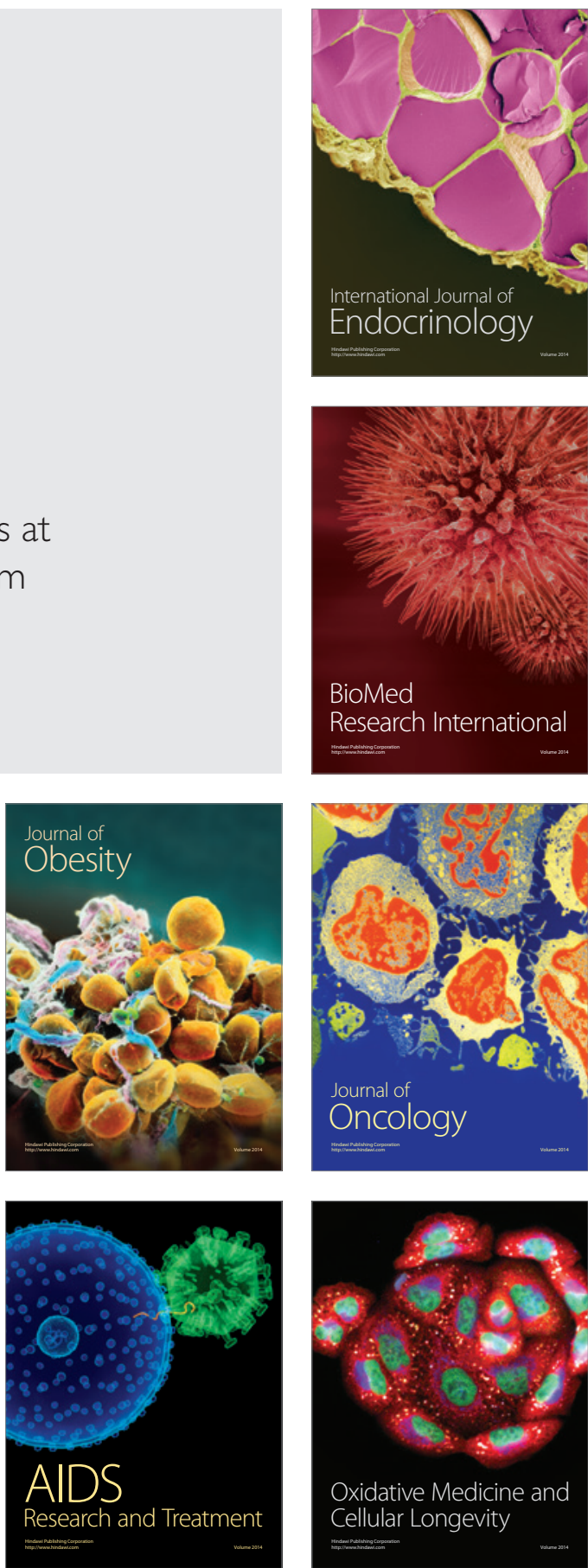